\title{
Multiobjective synchronization of coupled systems
}

\author{
Yang Tang, ${ }^{1, a)}$ Zidong Wang, ${ }^{2, b)}$ W. K. Wong, ${ }^{3, c)}$ Jürgen Kurths, ${ }^{4, d)}$ and Jian-an Fang ${ }^{5}$ \\ ${ }^{1}$ School of Information Science and Technology, Donghua University, Shanghai, China and Potsdam \\ Institute for Climate Impact Research, Potsdam, Germany \\ ${ }^{2}$ Department of Information Systems and Computing, Brunel University, Uxbridge, Middlesex, UB8 3PH, \\ United Kingdom \\ ${ }^{3}$ Institute of Textiles and Clothing, The Hong Kong Polytechnic University, Hong Kong, China \\ ${ }^{4}$ Potsdam Institute for Climate Impact Research, Potsdam, Germany and Institute of Physics, \\ Humboldt University, Berlin, Germany and Institute for Complex Systems and Mathematical Biology, \\ University of Aberdeen, Aberdeen AB24 3UE, United Kingdom \\ ${ }^{5}$ Department of Automation, Donghua University, Shanghai, China
}

(Received 31 January 2011; accepted 8 May 2011; published online 28 June 2011)

\begin{abstract}
In this paper, multiobjective synchronization of chaotic systems is investigated by especially simultaneously minimizing optimization of control cost and convergence speed. The coupling form and coupling strength are optimized by an improved multiobjective evolutionary approach that includes a hybrid chromosome representation. The hybrid encoding scheme combines binary representation with real number representation. The constraints on the coupling form are also considered by converting the multiobjective synchronization into a multiobjective constraint problem. In addition, the performances of the adaptive learning method and non-dominated sorting genetic algorithm-II as well as the effectiveness and contributions of the proposed approach are analyzed and validated through the Rössler system in a chaotic or hyperchaotic regime and delayed chaotic neural networks. ( 2011 American Institute of Physics. [doi:10.1063/1.3595701]
\end{abstract}

Synchronization of coupled chaotic systems has been a subject of great interest and importance, in theory but also various fields of application, such as secure communication and neuroscience. Recently, based on stability theory, synchronization of coupled chaotic systems by designing appropriate coupling has been widely investigated. However, almost all the available results have been focusing on ensuring the synchronization of coupled chaotic systems with as small coupling strengths as possible. In this contribution, we study multiobjective synchronization of coupled chaotic systems by considering two objectives in parallel, i. e., minimizing optimization of coupling strength and convergence speed. The coupling form and coupling strength are optimized by an improved multiobjective evolutionary approach. The constraints on the coupling form are also investigated by formulating the problem into a multiobjective constraint problem. We find that the proposed evolutionary method can outperform conventional adaptive strategy in several respects. The results presented in this paper can be extended into nonlinear time-series analysis, synchronization of complex networks and have various applications.

\footnotetext{
a) Also at Institute of Textiles and Clothing, The Hong Kong Polytechnic University, Hong Kong, China. Electronic mail: tangtany@gmail.com.

b) Also at School of Information Science and Technology, Donghua University, Shanghai, China. Electronic mail: Zidong.Wang@brunel.ac.uk.

${ }^{c)}$ Electronic mail: tcwongca@inet.polyu.edu.hk.

d)Electronic mail: Juergen.Kurths@pik-potsdam.de.
}

\section{INTRODUCTION}

Synchronization is widely observed in many fields such as chaotic systems, ${ }^{1-6}$ neural systems, ${ }^{7,8}$ and complex networks. ${ }^{9-12}$ In coupled chaotic oscillators, it is well-known that stability of the synchronized solution of coupled dynamical systems depends on the strength of the coupling (interaction or connection). ${ }^{13,14}$ One of the most intuitive approach dealing with synchronization of coupled chaotic systems is to use adaptive evolving coupling, which is based on feedback information and observed in many real-world networks. ${ }^{15-20}$ Although the importance of synchronization has been widely recognized, almost all available results have been focusing on ensuring the synchronization of coupled chaotic systems with as small coupling strengths as possible. In reality, however, the choice of coupling strengths actually affects the convergence rate and the coupling strengths which can be used to measure the synchronization performance or synchronization cost. To be more specific, in the process of designing coupling strengths, it is often essential that synchronization of chaotic systems is achieved with a convergence rate as quick as possible, while the control cost should be as small as possible. Therefore, it is meaningful to investigate the issue of synchronization of chaotic systems when simultaneously optimizing two conflicting objectives, i. e., cost of coupling strengths and convergence rate at the same time. In this paper, this kind of synchronization is called multiobjective synchronization, which can be regarded as a typical multiobjective optimization problem. A natural question arises: do there exist other coupling strengths which outperform adaptive strategies in terms of both convergence speed and control cost? Unfortunately, a literature 
search has revealed that such an issue has not yet been addressed, and the main reason lies in how to properly define objective functions and how to solve this problem, despite its importance in both theoretical and real-world applications. It is, therefore, the first motivation of this paper to address such a gap by making one of the first attempts to deal with multiobjective synchronization problem for a class of chaotic systems.

The limitations of control under constraints have been investigated in the control theory literature, see Refs. 21-23 and the references therein. For example, in Ref. 22, a class of controlled synchronization systems under information constraints imposed by limited information capacity of the coupling channel is analyzed. In practical situations, some dimensions in coupled systems should not be controlled due to a reduction of control cost or implementation constraints. In other words, it is essential to synchronize chaotic systems by inputting feedback coupling strengths locally on a small fraction of dimensions and exploiting the coupling effects to achieve synchronization. This type of control technique is called pinning control, which is widely used in many chaotic synchronization and network control problems. ${ }^{24,25}$ In Ref. 26, it is shown that master-slave synchronization of Lü systems can be ensured by only inputting one controller, which efficiently reduces the cost of control implementation. Up to now, the synchronization problem for chaotic systems with automatically selecting controlling dimensions has not been adequately addressed, which is another incentive for this research.

As a multiobjective optimization problem, one of the most popular ways to solve the multiobjective synchronization problem is to construct a single aggregate objective function. ${ }^{27}$ An easy and well-known combination is the weighted linear sum of the objectives. One has to specify scalar weights for each objective to be optimized a priori, and then to combine them into a single function that can be solved by any single-objective optimizer. Thus, the solution obtained in such a way will largely depend on the values (more precisely, the relative values) of the weights assigned. It should also be noted that the weighted sum method is essentially subjective, in that a decision maker needs to supply the weights. This approach has another limitation that only solutions located on the convex part of the Pareto front (PF) can be detected, i.e., one cannot identify all non-dominated solutions.

Evolutionary algorithms are a class of stochastic search heuristics that attempts to mimic biological processes of evolution, incorporating concepts of selection, reproduction, and mutation. ${ }^{27,28}$ Evolutionary algorithms have been widely used in synchronization of complex networks, ${ }^{10}$ PID control, ${ }^{29}$ dimensionality reduction, ${ }^{30}$ and designing neural networks, ${ }^{31}$ etc. Evolutionary algorithms are very popular approaches in multiobjective optimization. Nowadays, most evolutionary optimizers utilize Pareto-based ranking schemes. Genetic algorithms, such as the non-dominated sorting genetic algorithm-II $(\text { NSGA-II) })^{32}$ and strength Pareto evolutionary approach-2 (SPEA-2), ${ }^{33}$ have become standard approaches. An objective way of solving multiobjective problems requires a Pareto-compliant ranking method, favoring non-dominated solutions, as seen in current multiobjective evolutionary approaches such as NSGA-II and SPEA-2. Specially, in NSGA-II, the computational complexity is reduced and the elitism approach is adopted. In addition, no weight is required and thus no $a$ priori information on the problem is needed. However, in tackling the problem of multiobjective synchronization of chaotic systems, the real coded NSGA-II utilizing the simulated binary crossover (SBX) operator and polynomial mutation will lead to a slow convergence speed of approximating true Pareto front, since the decision variables in this multiobjective synchronization problem lie in both a discrete space and a continuous space, which contributes the third motivation of our research.

Motivated by the above discussion, a modified non-dominated sorting genetic algorithm-II (MNSGA-II) is proposed by a hybrid encoding scheme first in this paper. The problem of multiobjective synchronization of chaotic systems by optimizing both convergence speed and control cost is investigated in this paper. Multiobjective synchronization with constraints is also analyzed. It was shown from the experiments in Sec. IV C that the MNSGA-II outperformed the well-known NSGA-II in terms of convergence speed and distribution of the solutions. It is also verified that the solutions obtained by MNSGA-II dominated the solutions obtained by adaptive coupling.

The main contributions of this paper will be as follows:

(1) The problem of multiobjective synchronization of chaotic systems in terms of control cost and convergence speed is considered for the first time and solved by an MNSGA-II algorithm.

(2) In dealing with the problem of multiobjective synchronization of chaotic systems, not only the coupling strength, but also the coupling form is taken into consideration, which is converted into a constraint multiobjective problem.

(3) The MNSGA-II algorithm has a faster convergence speed to approach Pareto front than the NSGA-II algorithm does.

(4) The solutions obtained by MNSGA-II can dominate the solutions obtained by the adaptive feedback learning method in the simulation examples, and MNSGA-II can also provide more solutions for synchronization.

The remainder of this paper is organized as follows. In Sec. II, some preliminaries of the multiobjective synchronization problem are briefly outlined. In Sec. III, the encoding scheme of MNSGA-II is presented. In Sec. IV, the feasibility and contributions of the proposed approach are analyzed. Concluding remarks are given in Sec. V.

\section{MULTIOBJECTIVE SYNCHRONIZATION OF CHAOTIC SYSTEMS}

\section{A. Preliminaries}

Throughout this paper, $\delta_{\mathcal{D}}(\cdot)$ denotes the characteristic function of the finite set $\mathcal{D}$, i.e., $\delta_{\mathcal{D}}(i)=1$ if $i \in \mathcal{D}$; otherwise, $\delta_{\mathcal{D}}(i)=0$.

In this paper, to illustrate the multiobjective synchronization of chaotic systems, the following systems are considered for the sender and receiver (or master and slave),

$$
\begin{aligned}
\dot{x}(t) & =f(x(t), x(t-\tau(t))), \\
\dot{y}(t) & =f(y(t), y(t-\tau(t)))+\alpha[g(x(t))-g(y(t))],
\end{aligned}
$$

where $x(t)=\left[x_{1}, \ldots, x_{n}\right]$ is the $n$-dimensional state of the sender system and $y(t)=\left[y_{1}, \ldots, y_{n}\right]$ is the $n$-dimensional 
state of the receiver system; $\tau(t)$ is a time-varying delay for delayed chaotic systems; $f(\cdot)$ is the dynamics of an uncoupled system and supposed to be chaotic, $f: \mathbb{R}^{n} \rightarrow \mathbb{R}^{n}$; $\alpha=\operatorname{diag}\left(c_{1}, \ldots, c_{n}\right) \in \mathbb{R}^{n \times n}$ is a diagonal matrix representing the coupling strength; $g(\cdot)$ is an output function, $g: \mathbb{R}^{n} \rightarrow \mathbb{R}^{n}$. The output function $g(x(t))$ can be rewritten as $g(x(t))=\Gamma x(t)$, where $\Gamma=\operatorname{diag}\left(\delta_{\mathcal{D}}(1), \delta_{\mathcal{D}}(2), \ldots, \delta_{\mathcal{D}}(n)\right)$ is a diagonal inner coupling. In many practical situations, communication between the connected systems involves only a subset of the dynamical state variables of the systems and the communication channel might suffer from constraints. In this paper, the selection of $\Gamma$ is automatically implemented by a genetic algorithm. Three cases considered are as follows:

(1) Selection of $\Gamma$ without any constraint;

(2) Only one dimension is connected $\left(\sum_{i}^{n} \delta_{\mathcal{D}}(i)=1\right.$, $i=1,2, \ldots, n)$; and

(3) One dimension cannot be controlled $\left(\delta_{\mathcal{D}}(i) \neq 1\right.$, $i=1,2, \ldots, n)$.

Therefore, the second part of Eq. (1) can be rewritten as,

$$
\dot{y}(t)=f(y(t), y(t-\tau(t)))+K[x(t)-y(t)],
$$

where $K=\operatorname{diag}\left(\delta_{\mathcal{D}}(1) c_{1}, \ldots, \delta_{\mathcal{D}}(n) c_{n}\right)$, in which $c_{i}(i=1$, $\ldots, n)$ is the coupling strength.

For the adaptive coupling, in this paper, we consider the following adaptive strategy:

$$
\dot{y}(t)=f(y(t), y(t-\tau(t)))+\mathcal{K}[x(t)-y(t)],
$$

where $\mathcal{K}=\operatorname{diag}\left(\delta_{\mathcal{D}}(1) c_{1}, \ldots, \delta_{\mathcal{D}}(n) c_{n}\right)$ is updated according to the following laws:

$$
\begin{cases}\dot{c}_{i}=\mu\left(x_{i}(t)-y_{i}(t)\right)^{2}, & \delta_{\mathcal{D}}(i)=1, \\ c_{i}=0, & \delta_{\mathcal{D}}(i)=0,\end{cases}
$$

where $\mu>0$ is an arbitrary constant.

In order to optimize the control cost and the convergence speed, we introduce the average synchronization error $E$ and the average control $\operatorname{cost} C$ as follows:

$$
\begin{gathered}
E=\frac{1}{t_{2}-t_{1}} \int_{t_{1}}^{t_{2}}\|x(t)-y(t)\| d t, \\
\left\{\begin{array}{l}
C=\frac{1}{t_{2}-t_{1}} \int_{t_{1}}^{t_{2}}\|K\| d t, \text { for Eq. (2), } \\
C=\frac{1}{t_{2}-t_{1}} \int_{t_{1}}^{t_{2}}\|\mathcal{K}\| d t, \text { for Eq. (3). }
\end{array}\right.
\end{gathered}
$$

From the above two quantities, it is observed that the smaller the $E$ and $C$, the better the control performance. In this paper, our goal is to obtain solutions which make $E$ and $C$ as small as possible at the same time.

In the field of optimal control, one can formulate the following equation:

$$
R=\int_{0}^{\infty}\left(e^{T}(t) P_{1} e(t)+u^{T}(t) P_{2} u(t)\right)
$$

where $e(t)=x(t)-y(t), u(t)=\mathcal{K}(x(t)-y(t)), P_{1}$ and $P_{2}$ are two positive matrices. Here, $R$ is a combination of (5) and
(6). First, in order to minimize $R, P_{1}$ and $P_{2}$ should be provided in advance. As discussed in the Sec. I, the solution obtained in such a way will largely depend on the values of the weights assigned. Second, the system is a nonlinear one and it is usually impossible to obtain an analytic solution. Finally, even if a solution can be obtained, it is very difficult to avoid unavoidable conservativeness due to the existing mathematical assumptions.

\section{B. Multiobjective optimization}

At present, many practical applications involve complex optimization problems with various objectives that are often noncommensurable and conflicting in nature. The variety of objectives, together with the presence of numerous constraints, leads to the difficulty of tackling such problems, if not infeasible to solve without the support of powerful and efficient optimization algorithms. Here, without any loss of generality, a minimization problem with a decision space $\Omega$ is considered, since $E$ and $C$ should be minimized to a value as small as possible. For the minimization problem, we intend to solve for a variable set $V$ that optimizes the following objective:

$$
\min _{V \in \Omega} F(V), V \in \mathbb{R}^{n},
$$

where $V=\left\{v_{1}, v_{2}, \ldots, v_{n}\right\}$ is a vector with a set of decision variables and $F=\left\{f_{1}, f_{2}, \ldots, f_{m}\right\}$ is the objective vector with $m$ objectives to be minimized.

The following concepts of Pareto dominance and Pareto optimality are fundamental in multiobjective optimization, with Pareto dominance forming the basis of solution quality.

Definition 1 (Pareto Dominance): Given the objective vectors $Y_{1} \in \mathbb{R}^{m}$ and $Y_{2} \in \mathbb{R}^{m}$, then $\mathrm{Y}_{1}$ dominates $\mathrm{Y}_{2}$, denoted as $Y_{1} \prec Y_{2}$, iff $y_{1 i} \leq y_{2 i}, \forall i \in\{1,2, \ldots, m\}$, and $y_{1 i}<y_{2 i}, \exists \mathrm{i} \in\{1,2, \ldots, \mathrm{m}\}$.

Definition 2 (Optimal Pareto Front): The optimal Pareto front $(\mathrm{PF})$ denoted by $\mathcal{F}^{*}$ is the set of individuals,

$$
\mathcal{F}^{*}=\left\{\mathcal{F}_{j}^{*} \mid \mathcal{F}_{j}^{*} \prec \mathcal{F}_{i}, \forall \mathcal{F}_{i} \in \mathcal{F}\right\} .
$$

Different from single objective optimization, the solution to the multiobjective optimization problem exists in the form of alternate tradeoffs known as optimal Pareto optimal set. The different dominance relationship is illustrated in Fig. 1, where the solutions denoted by red circles form the Pareto front and dominate the solutions represented by blue square circles. It should be noted that each objective component of any nondominated solution in the Pareto optimal set can only be improved by degrading at least one of its other objective components. ${ }^{32}$

In this paper, evolutions of both the inner coupling $\Gamma$ and the coupling strengths $\alpha$ are considered. This problem is distinguished from previous work as it is regarded as a multiobjective problem where the two objectives (e.g., Eqs. (5) and (6)] of control cost $C$ and synchronization error $E$ are conflicting in nature. In order to ensure synchronization and make the designed coupling strength not very large, the constraints are placed on $E$ and $C$. As discussed in Sec. II A, the 


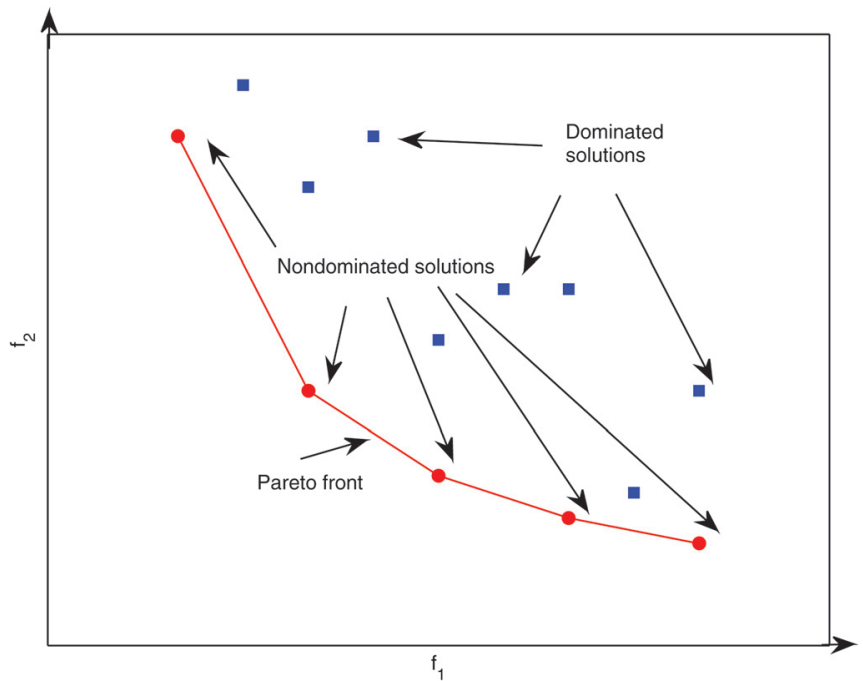

FIG. 1. (Color online) Illustration of the optimal Pareto front and the relationship between dominated and nondominated solutions.

first case of the optimization problem for the multiobjective synchronization of chaotic systems can be given as:

$$
\begin{array}{r}
f_{1}=\min \{C\}, \\
f_{2}=\min \{E\}, \\
\text { s.t. } E<\xi_{e}, C<\xi_{c},
\end{array}
$$

where $\xi_{e}$ and $\xi_{c}$ are constant. The upper bounds of $\xi_{e}$ and $\xi_{c}$ might be determined by an adaptive coupling method, which is similar to the reference point based method in the multiobjective problem. On the other hand, as pointed out in Sec. II A, communication between the connected systems involves a small fraction of the dynamical state variables of the systems and the communication channel might suffer from constraints. Therefore, communication constraints are also considered in this paper. The second problem is that only one dimension in the chaotic system is coupled. The problem can be formulated as follows:

$$
\begin{aligned}
& \qquad f_{1}=\min \{C\}, \\
& f_{2}=\min \{E\}, \\
& \text { s.t. } \quad E<\xi_{e}, C<\xi_{c}, \sum_{i}^{n} \delta_{\mathcal{D}}(i)=1 .
\end{aligned}
$$

The third problem is that one dimension should not be coupled. The problem can be written as:

$$
\begin{aligned}
& \quad f_{1}=\min \{C\}, \\
& f_{2}=\min \{E\}, \\
& \text { s.t. } \quad E<\xi_{e}, C<\xi_{c}, \delta_{\mathcal{D}}(i) \neq 1 .
\end{aligned}
$$

The second and the third problems can also be regarded as pinning control, which means only a fraction of dimensions is controlled. Note that there exist $2^{n}-1$ possibilities of control dimensions. When $n$ exceeds 20 , there exist at least 1048575 choices. It is hard to tackle the problem of selection schemes using the enumeration method. On the other hand, there exists no literature to solve the problem of selecting control dimensions efficiently. In this paper, the selection of controlling dimensions is automatically selected by evolutionary algorithms.

\section{A MODIFIED NSGA-II}

Conventional approaches to multiobjective optimization typically transform the original problem into a single optimization problem and use point-by-point algorithms to iteratively find a better solution. Limitations of such approaches are that the multiobjective problem should be well-behaved, i.e., differentiability or satisfying the Kuhn-Tucker conditions, the assigned weights of various objectives and the generation of only one solution for each simulation run. Recently, metaheuristical methods that are inspired by biological or multiagent phenomena such as evolutionary algorithms and particle swarm optimization have been gaining increasing attention as a much more flexible and effective candidate to dealing with complex optimization problems. Among these meta-heuristics, multiobjective evolutionary algorithm is a stochastic search methodology to solve multiobjective problems involving multiple noncommensurable and competing criteria. Through mimicking the Darwin-Wallace principle of survival-of-the-fittest, multiobjective evolutionary algorithms have the distinct advantage of being able to sample multiple solutions simultaneously. With such a feature, multiobjective evolutionary algorithms have the capability of dealing with the multiobjective problem as well as finding nondominated sets in a single run.

Owing to the popularity and efficiency of NSGA-II, it is used to solve the multiobjective synchronization problem of chaotic systems. NSGA-II includes diversity preservation, nondominated sorting approach, and elitism method. The function of the elitism method is that the best solution of the population in each step is reserved and used for mutation and crossover for the next step. As a real-coded NSGA-II algorithm is able to find a better spread of solutions than a binary-coded NSGA-II, while a real-coded NSGA-II provides similar results to appropriate true optimal PF with a binarycoded NSGA-II, ${ }^{32}$ the real-coded NSGA-II is used in this paper. The real-coded NSGA-II includes a simulated binary crossover and polynomial mutation operators. The SBX operator simulates the working principle of the single-point crossover operator on binary strings. It has been verified that SBX respects the interval schemata processing, in the sense that common interval schemata between parents are preserved in children. It should be also mentioned that more details of jargons such as elitism approach and SBX in the evolutionary algorithm can be found in Refs. 27, 28, and 32. As problems studied in this paper are under constraints, the following constrained dominance is employed to tackle the constrained problem: ${ }^{32}$

A solution $i$ is said to constrained-dominate a solution $j$, if any of the following conditions is true:

(1) Solution $i$ is feasible but solution $j$ is not.

(2) Solutions $i$ and $j$ are both infeasible, but solution $i$ has a smaller overall constraint violation.

(3) Solutions $i$ and $j$ are feasible and solution $i$ dominates solution $j$. 
The effect of using this constrained-domination principle is that any feasible solution has a better nondomination rank than any infeasible solution. All feasible solutions are ranked according to their nondomination level based on the objective function values. Among two infeasible solutions, the solution with a smaller constraint violation has a better rank.

A detailed implementation of MNSGA-II is introduced as follows. MNSGA-II is a kind of genetic algorithm (GA), which is a search heuristic that simulates the process of natural evolution. This heuristic is routinely used to generate useful solutions to optimization and search problems. GAs generate solutions to optimization problems using techniques inspired by natural evolution, such as mutation, selection, and crossover. In a GA, a population of strings (called chromosomes), which encode candidate solutions (called individuals) to an optimization problem, evolves toward better solutions. Each dimension in chromosomes is called a gene. The first step of the MNSGA-II is to encode possible control solutions into chromosomes. A chromosome represents a feasible solution. To handle the synchronization problem addressed, a feasible solution should be able to determine the assignment of control dimension and control gain. In this research, each chromosome is a sequence of genes whose length is equal to two times of the chaotic dimension $n$. The encoding scheme is shown in Fig. 2. As seen in Fig. 2, the encoding scheme includes two parts. Each gene in part A identifies the activation of dimension $i$ and the value of each gene in part $\mathrm{B}$ indicates control gain in each dimension $i$ of chaotic systems. For original real-coded NSGA-II, part A is a continuous space and is used to control the activation of dimension $i$, that is, the controller in dimension $i$ is activated according to the value of $a_{i}$. Part B is the corresponding control gain of Part A. For real-coded NSGA-II, if $a_{i}>0.5$, $\delta_{\mathcal{D}}(i)=1$, i.e., the dimension $i$ is controlled by the control gain $c_{i}$.

Although NSGA-II can deliver good performance and high efficiency in dealing with multiobjective optimization, NSGA-II might not be suitable to solve the multiobjective synchronization of chaotic systems, since both discrete and continuous decision spaces are included. If part $\mathrm{A}$ of each chromosome is evolved using SBX and polynomial mutation, the switching between different selections of $\delta_{\mathcal{D}}(i)$ is very slow, which might lead to a slow convergence speed. Therefore, an MNSGA-II is proposed here. Different from the original NSGA-II, part A of each chromosome is a discrete coding scheme. A binary encoding scheme is utilized here. If $a_{i}=1$, the dimension $i$ is controlled and the control gain $c_{i}$ is used to control. If $a_{i}=0$, the dimension $i$ is not con-

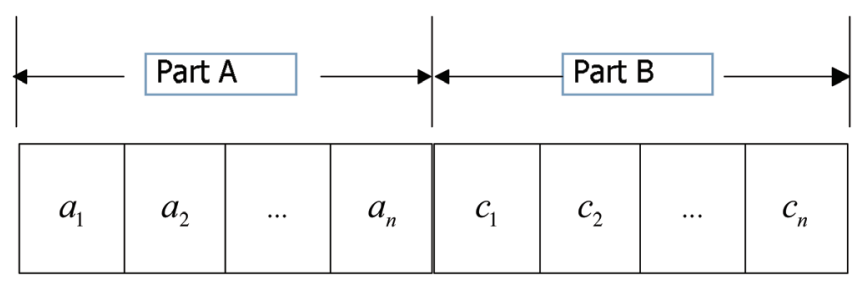

FIG. 2. (Color online) Encoding scheme of multiobjective synchronization. trolled. Part B in MNSGA-II is the same as the original NSGA-II. The chromosomes in the population will benefit from this mechanism and have the ability of fast convergence speed.

Since both discrete and continuous spaces are considered in MNSGA-II, a hybrid crossover and mutation scheme is adopted in this paper. For part B of the encoding scheme, the SBX and polynomial mutation are employed, which are the same as NSGA-II. For part A of the encoding scheme, to adapt the proposed presentation, a crossover operator is adopted based on the uniform crossover and mutation, ${ }^{28}$ which is implemented by using the following five steps:

(1) Randomize a bit string with the same length as the chromosomes;

(2) Find the gene positions where the value is 1 (or 0 ) in the bit string;

(3) Fill in the same positions in child 1 (or child 2) by copying the genes from the gene positions of parent 1 (or parent 2) found in step 2;

(4) Fill in the remaining positions in child 1 (or child 2) by copying the genes from the other gene positions of parent 2 (or parent 1 );

(5) Check if the generated child chromosome is an invalid solution. If so, go to step 1; otherwise output the generated chromosomes.

In this study, a mutation operator is used based on the uniform mutation, ${ }^{8}$ which is implemented according to the steps below:

(1) Randomly generate a positive integer $r[r \in(1, n)]$.

(2) Randomly select $r$ genes as mutation genes in the original chromosome;

(3) For each mutation genes selected, randomly change its value;

(4) Check whether the chromosome generated in step 3 is an invalid solution. If so, go to step 2; otherwise output the generated chromosome.

Note that if the control dimension can be fixed a prioi, part A of each chromosome can be removed and part B of each chromosome remains. Only the control gains are designed to synchronize the chaotic systems.

\section{NUMERICAL EXAMPLES}

This section shows that the MNSGA-II algorithm not only outperforms the conventional adaptive coupling method, but also delivers better performance than the original NSGA-II algorithm. Three different paradigms, i. e., three-order Rössler system, ${ }^{34}$ delayed chaotic neural networks $^{35}$ (DCNN), and hyperchaotic Rössler system ${ }^{36}$ are used to carry out numerical examples. Three kinds of problems introduced in Sec. II are investigated in this research.

\section{A. Chaotic systems}

First, three chaotic systems including Rössler system, delayed chaotic neural networks, and hyperchaotic Rössler system are introduced. The Rössler system is given by the following differential equations: 
TABLE I. The initial values of sender (IVS) and initial values of receiver (IVR).

\begin{tabular}{lcc}
\hline \hline System & IVS & IVR \\
\hline Rössler system & $10,10,10$ & $0.3,0.5,0.7$ \\
DCNNs & 1,1 & $0.3,0.5$ \\
Hyperchaotic system & $-10,-6,0,10$ & $-10.5,-6.5,0.5,10.5$ \\
\hline \hline
\end{tabular}

$$
\left\{\begin{array}{l}
\dot{x}_{1}=-x_{2}-x_{3}, \\
\dot{x}_{2}=x_{1}+a x_{2}, \\
\dot{x}_{3}=b+x_{3}\left(x_{1}-c\right),
\end{array}\right.
$$

where $a, b$, and $c$ are constants. In this study, $a=0.2$, $b=0.2$, and $c=10$ are used. The Rössler system then displays chaotic behavior. Next, the following delayed chaotic neural network is considered here:

$$
\left\{\begin{aligned}
\dot{x}_{1}= & -x_{1}+1.8 \tanh \left(x_{1}\right)-0.15 \tanh \left(x_{2}\right) \\
& +1.7 \tanh \left(x_{1}(t-\tau(t))\right) \\
& -0.12 \tanh \left(x_{2}(t-\tau(t))\right) \\
\dot{x}_{2}= & -x_{2}-5.2 \tanh \left(x_{1}\right)+3.5 \tanh \left(x_{2}\right) \\
& -0.26 \tanh \left(x_{1}(t-\tau(t))\right) \\
& -2.5 \tanh \left(x_{2}(t-\tau(t))\right)
\end{aligned}\right.
$$

where $\tau(t)=\frac{e^{t}}{e^{t}+1}$ is a time-varying delay. Finally, a hyperchaotic Rössler system is studied,

$$
\left\{\begin{array}{l}
\dot{x}_{1}=-x_{2}-x_{3}, \\
\dot{x}_{2}=x_{1}+a x_{2}+x_{4}, \\
\dot{x}_{3}=b+x_{1} x_{3} \\
\dot{x}_{4}=-c x_{3}+d x_{4}
\end{array}\right.
$$

where $a, b, c$, and $d$ are constants. Here, $a=0.25, b=3$, $c=0.5$, and $d=0.05$ are used here.

The initial values of sender (IVS) and initial values of receiver (IVR) of three chaotic systems are listed in Table I. Note that only one set of IVS and IVR is provided in this paper. However, different IVS and IVR will result in the similar results. In this paper, due to the sake of simplicity, the representative results are presented.

\section{B. Comparison of adaptive coupling and MNSGA-II}

Here, the well-known adaptive strategy and MNSGA-II are compared with or without restrictions on $\Gamma$.

TABLE II. Synchronization of the Rössler system with different $\Gamma$ using adaptive coupling and MNSGA-II.

\begin{tabular}{lcc}
\hline \hline$\Gamma$ & Symbols for AC & Symbols for MNGA-II \\
\hline$\{1,0,0\}$ & $A_{1}$ & \\
$\{0,1,0\}$ & $A_{2}$ & Control one dimension \\
$\{0,0,1\}$ & $A_{3}$ & \\
$\{1,1,0\}$ & $B_{1}$ & Control two dimensions \\
$\{1,0,1\}$ & $B_{2}$ & \\
$\{0,1,1\}$ & $B_{3}$ & Control three dimensions \\
$\{1,1,1\}$ & $C_{1}$ & \\
\hline \hline
\end{tabular}

TABLE III. Synchronization of DCNNs with different $\Gamma$ using adaptive coupling and MNSGA-II.

\begin{tabular}{lcc}
\hline \hline$\Gamma$ & Symbols for AC & Symbols for MNGA-II \\
\hline$\{1,0\}$ & $A_{1}$ & Case I \\
$\{0,1\}$ & $A_{2}$ & Case II \\
$\{1,1\}$ & $B_{1}$ & Case III \\
\hline \hline
\end{tabular}

\section{Comparison of adaptive coupling and MNSGA-II with unconstrained $\Gamma$}

Three examples with constraints on $E$ and $C$ are provided and the restriction on $\Gamma$ is not considered in this subsection. In order to compare the well-known adaptive strategy with our MNSGA-II, we enumerate all the cases of $\Gamma$ for couplings and use the adaptive schemes to update the coupling strengths. Details of $\Gamma$ are shown in Tables II-IV.

The Runge-Kutta method with a stepsize 0.01 is used to simulate all the models. If there is no further statement, $t_{1}=0$ and $t_{2}=20$ are adopted. The initial values of adaptive strength are 0 for all the dimensions of different chaotic systems. The constant $\mu$ is taken as $\mu=10$ in the adaptive strategy. Note that a large $\mu$ will result in a large $E$ with a small $C$ and vice visa. We have tested different $\mu$ and similar results can be obtained. Here, the representative results are provided. If without any further statement, for MNSGA-II, the population size is 100 and the generation number is $T=250$. For SBX and polynomial mutation, the crossover probability of $p_{c}=0.9$ and a mutation probability of $p_{m}=0.1$ are used. ${ }^{32}$ We use distribution indexes for crossover and mutation operators as $\eta_{c}=20$ and $\eta_{m}=20$, (Ref. 27), respectively. The search space of coupling strength in all the dimensions is set $[0,50]$. The population obtained at the end of $T$ generations (the population after elite-preserving operator is applied) is used to calculate a couple of performance metrics $(E$ and $C$ ).

In order to demonstrate the effectiveness of the MNSGA-II and the adaptive strategy, we show typical

TABLE IV. Synchronization of the hyperchaotic Rössler system with different $\Gamma$ using adaptive coupling and MNSGA-II.

\begin{tabular}{lcc}
\hline \hline$\Gamma$ & Symbols for AC & Symbols for MNGA-II \\
\hline$\{1,0,0,0\}$ & $A_{1}$ & \\
$\{0,1,0,0\}$ & $A_{2}$ & Control one dimension \\
$\{0,0,1,0\}$ & $A_{3}$ & \\
$\{0,0,0,1\}$ & $A_{4}$ & \\
$\{1,1,0,0\}$ & $B_{1}$ & \\
$\{1,0,1,0\}$ & $B_{2}$ & \\
$\{1,0,0,1\}$ & $B_{3}$ & \\
$\{0,1,1,0\}$ & $B_{4}$ & \\
$\{0,1,0,1\}$ & $B_{5}$ & \\
$\{0,0,1,1\}$ & $B_{6}$ & \\
$\{1,1,1,0\}$ & $C_{1}$ & \\
$\{1,1,0,1\}$ & $C_{2}$ & Control two dimensions \\
$\{1,0,1,1\}$ & $C_{3}$ & \\
$\{0,1,1,1\}$ & $C_{4}$ & \\
$\{1,1,1,1\}$ & $D_{1}$ & \\
\hline \hline
\end{tabular}




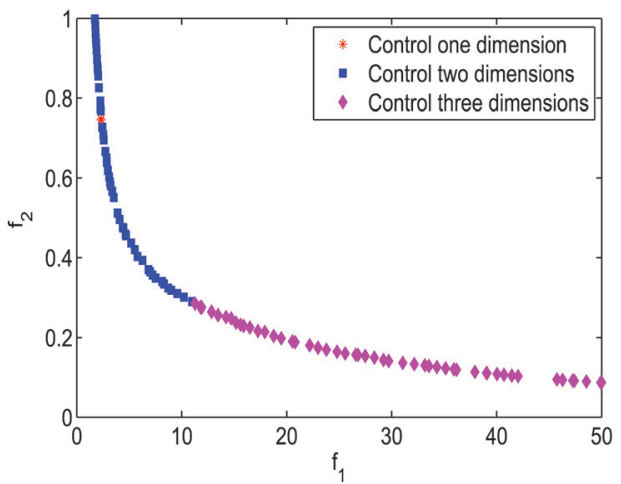

(a)

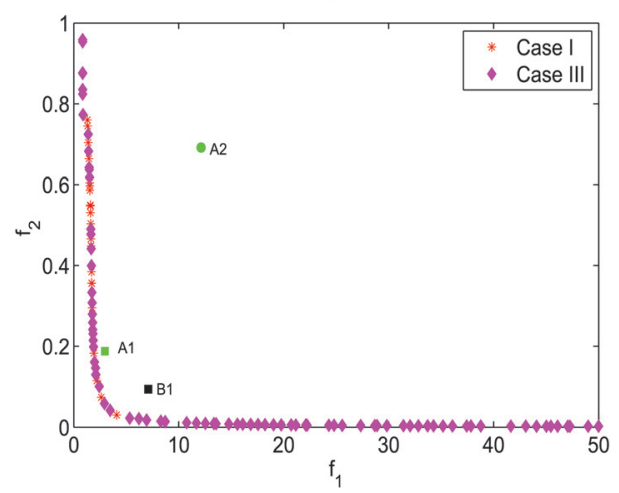

(c)

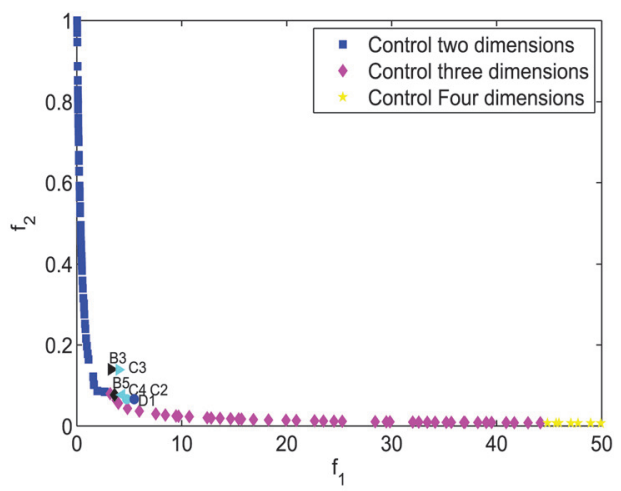

(e)

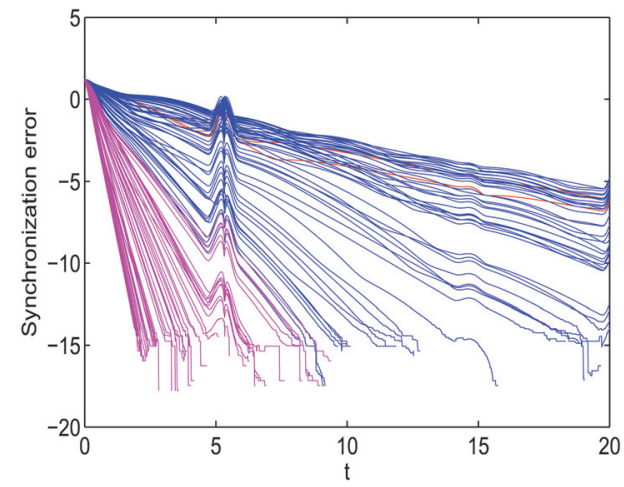

(b)

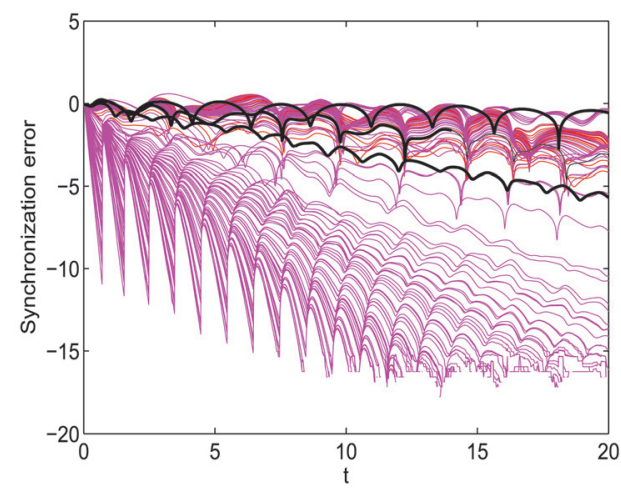

(d)

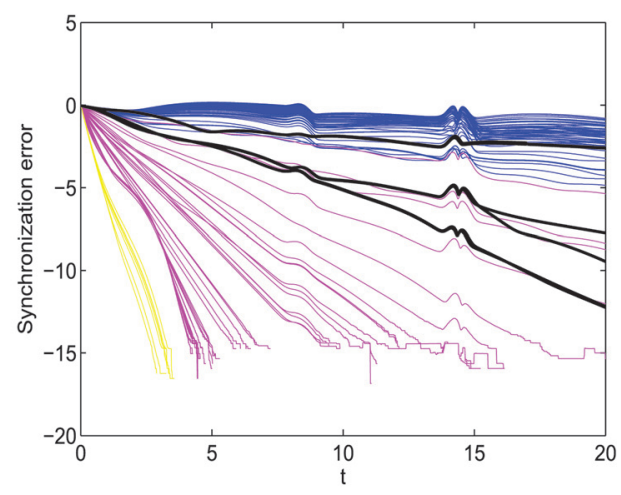

(f)
FIG. 3. (Color online) Synchronization errors of chaotic systems (errors plotted in $\log _{10}$ ) and solutions (Computing $f_{1}$ and $f_{2}$ ) obtained by adaptive strategy and MNSGA-II. In (b), (d), and (f), black lines are the errors by adaptive strategy; other color lines are the corresponding synchronization errors using the same solutions by MNSGA-II. (a) Solutions obtained by adaptive strategy and MNSGA-II for synchronization of the Rössler system; (b) synchronization errors of the Rössler system using adaptive strategy and MNSGA-II; (c) solutions obtained by adaptive strategy and MNSGA-II for synchronization of DCNN; (d) synchronization errors of DCNN using adaptive strategy and MNSGA-II (black lines are the errors by adaptive strategy); (e) solutions obtained by adaptive strategy and MNSGA-II for synchronization of the hyperchaotic Rössler system; (f) synchronization errors of the hyperchaotic Rössler system using adaptive strategy and MNSGA-II (black lines are the errors by adaptive strategy). simulation results of both MNSGA-II and adaptive strategies for the three chaotic systems. For the sake of simplicity and reality, the coupling strength should not be too large and the synchronization error should be small enough to ensure synchronization. We set constraints of $\xi_{E}=1$ and $\xi_{C}=50$ for the chaotic systems. Once the solutions obtained by MNSGA-II exceed the boundary, the solutions are regarded as infeasible solutions. One might wonder why such parameters are set. If we relax the bounds of $\xi_{E}$ and $\xi_{C}$, more infeasible (or impractical) solutions will be obtained (the coupling strengths are too large or synchronization of the chaotic systems cannot be guaranteed). One can also place more restrictions on $\xi_{E}$ and $\xi_{C}$; however, in order to show the diversity of solutions, we do not make $\xi_{E}$ and $\xi_{C}$ too small. In Figs. 3 and 4, the synchronization error is calculated as $\log _{10}\|x(t)-y(t)\|$.

Figures 3(a), 3(c), and 3(e) show the solutions obtained after 250 generations with MNSGA-II and the solutions obtained by the adaptive strategy. First, it can be observed from Figs. 3(a), 3(c), and 3(e) that the solutions obtained by MNSGA-II dominate all the solutions obtained by the adaptive strategy. For the Rössler system, we can see that only $A_{1}, A_{2}$, and $B_{1}$ obtained by the adaptive scheme are plotted in Fig. 3(a), which indicates that they are feasible solutions. It is also visible that the chaotic systems coupled (controlled) by one dimension will lead to a small control cost but a slow convergence rate. Controlling three dimensions of chaotic systems will result in a fast convergence speed but the control cost is usually very large. Controlling two dimensions of chaotic systems is an intermediate way among the three schemes. From Fig. 3(b), the results of synchronization errors confirm the above observations that a number of solutions obtained by MNSGA-II can achieve faster convergence rate for synchronization than by the adaptive strategy. The solutions obtained by controlling three dimensions with MNSGA-II can achieve the fastest convergence speed among the three coupling schemes. 

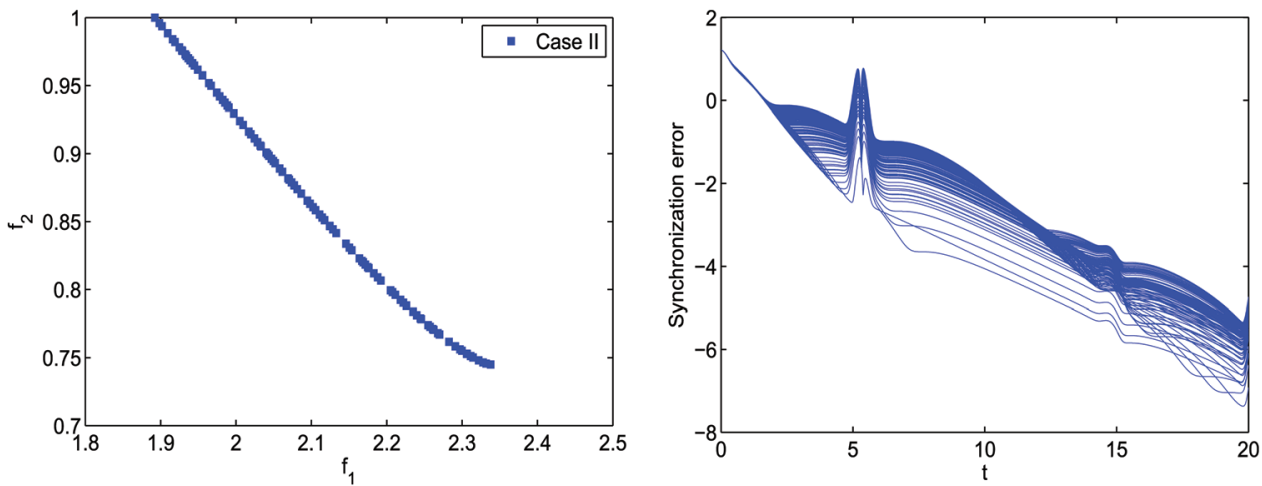

(a)

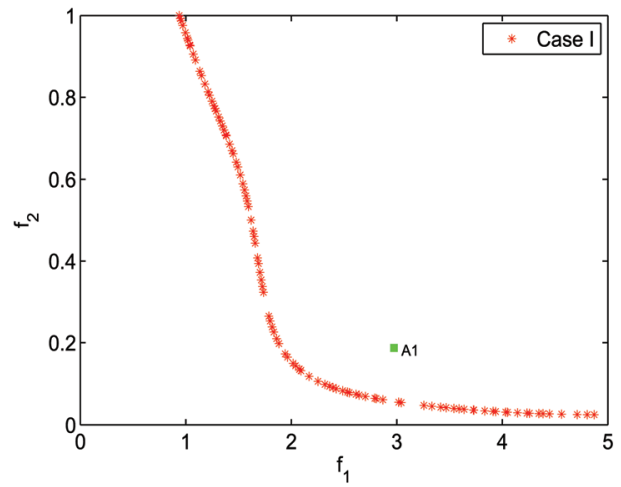

(c)

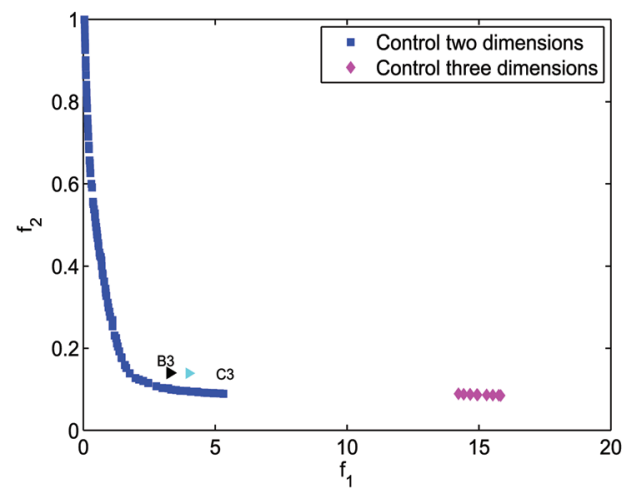

(e) (b)

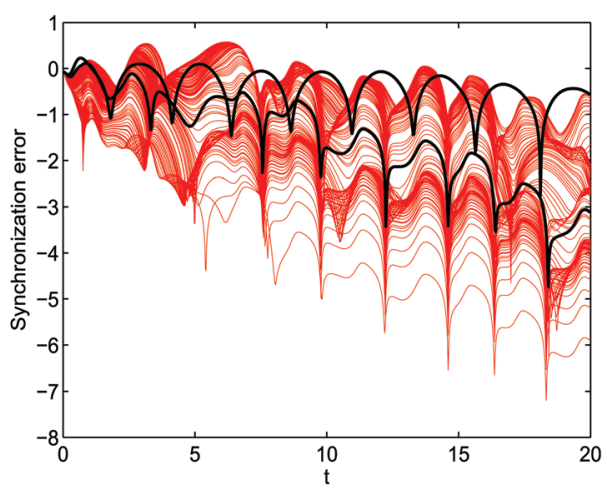

(d)

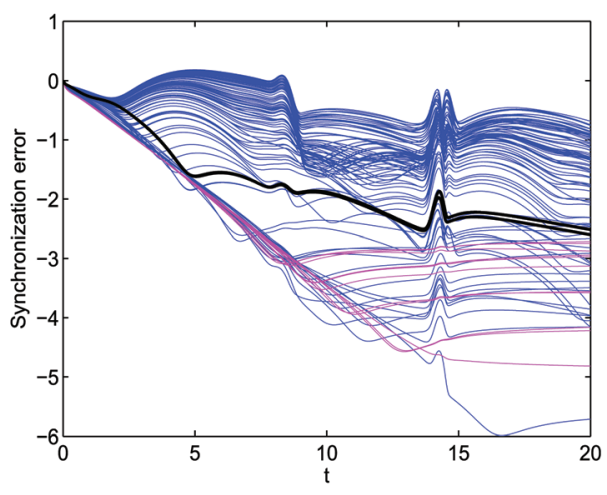

(f)
FIG. 4. (Color online) Synchronization errors of chaotic systems (errors plotted in $\log _{10}$ ) and solutions (Computing $f_{1}$ and $f_{2}$ ) obtained by adaptive strategy and MNSGA-II with constrained $\Gamma$. In (b), (d) and (f), black lines are the errors by adaptive strategy; other color lines are the corresponding synchronization errors using the same solutions by MNSGA-II. (a) Solutions obtained by adaptive strategy and MNSGA-II for synchronization of the Rössler system; (b) synchronization errors of the Rössler system using adaptive strategy and MNSGA-II; (c) solutions obtained by adaptive strategy and MNSGA-II for synchronization of DCNN; (d) synchronization errors of DCNN using adaptive strategy and MNSGA-II (black lines are the errors by adaptive strategy); (e) solutions obtained by adaptive strategy and MNSGA-II for synchronization of the hyperchaotic Rössler system; (f) synchronization errors of the hyperchaotic Rössler system using adaptive strategy and MNSGA-II (black lines are the errors by adaptive strategy).
For synchronization of DCNN, the results of solutions and synchronization errors are shown in Figs. 3(c) and 3(d). It can be observed that the solutions $A_{1}$ and $B_{1}$ by the adaptive scheme dominate the solution $A_{2}$. By MNSGA-II, we find that the solutions when $\Gamma=\{0,1\}$ are not shown in Fig. 3(c), which indicates that controlling the first dimension of DCNN is more efficient than controlling the second dimension. Such a phenomenon also confirms the results obtained by adaptive coupling. It can also be found that controlling two dimensions will lead to a faster convergence speed and a larger coupling strength than controlling one dimension. From Fig. 3(d), it was also found that a number of solutions of MNSGA-II can achieve faster convergence rate for synchronization than those of the adaptive strategy.

For synchronization of the hyperchaotic Rössler system, the results of solutions and synchronization errors are illustrated in Figs. 3(e) and 3(f). It is seen from Figs. 3(e) and 3(f) that the results of controlling one dimension are not shown, which mean that the results of controlling one dimen- sion are infeasible solutions or dominated by other solutions. It was found that as more dimensions are controlled, the smaller (quicker) the synchronization error $E$ and the larger the control cost $C$ become.

To summarize, the advantages of MNSGA-II over the adaptive scheme are listed as follows:

(1) Some solutions obtained by MNSGA-II dominate the solutions obtained by the adaptive coupling, which means that the convergence speed of the adaptive coupling can be further improved with a smaller control cost.

(2) MNSGA-II is able to provide much more solutions than the conventional adaptive coupling method does. One can select one of them, which is able to fit the circumstance implementation best.

(3) MNSGA-II has the ability of automatically finding efficient coupling dimensions under implementation constraints. 
TABLE V. Synchronization of the Rössler system with different $\Gamma$ using adaptive coupling and MNSGA-II.

\begin{tabular}{lcc}
\hline \hline$\Gamma$ & Symbols for AC & Symbols for MNGA-II \\
\hline$\{1,0,0\}$ & $A_{1}$ & Case I \\
$\{0,1,0\}$ & $A_{2}$ & Case II \\
$\{0,0,1\}$ & $A_{3}$ & Case III \\
\hline \hline
\end{tabular}

\section{Comparison of adaptive coupling and MNSGA-II with constrained $\Gamma$}

Now, synchronization of chaotic systems with constrained $\Gamma$ is investigated. For the Rössler system and DCNN, the second problem in Sec. II is studied, i.e., only one dimension is coupled. For the hyperchaotic Rössler system, the third problem in Sec. II is studied, i.e., only one dimension is not coupled. Here, we assume that the second dimension is not coupled. In order to compare the wellknown adaptive strategy with MNSGA-II, we enumerate all the cases of $\Gamma$ for coupling and employ the adaptive schemes to update the coupling strengths. The symbols are listed in Tables III-V.

The solutions obtained after 250 generations with MNSGA-II and the adaptive strategy are shown in Figs. 4(a), 4(c), and 4(e). Similar with the results in Sec. IV B 1, we find that the solutions obtained by MNSGA-II dominate the solutions obtained by the adaptive approach. For the Rössler system, it is shown from Fig. 4(a) that controlling the second dimension is the most efficient coupling way to achieve synchronization. The solutions obtained by the adaptive approach are infeasible solutions and hence are not plotted in Figs. 4(a) and (b). For DCNN, controlling the second dimension is more powerful than controlling the first dimension, as seen from Figs. 4(c) and (d). One reason might explain this phenomenon is that the second dimension $x_{2}$ has larger coupling with the first dimension $x_{1}$ than the first dimension $x_{1}$ does. For the hyperchaotic Rössler system, only two solutions $B_{3}$ and $C_{3}$ obtained by the adaptive scheme are feasible. However, $B_{3}$ and $C_{3}$ are also dominated by the solutions obtained by MNSGA-II. From Figs. 4(b), 4(d), and 4(f), the results indicate that a number of solutions obtained by MNSGA-II have faster convergence speed to achieve synchronization than the solutions obtained by the adaptive strategy.

\section{Comparison of NSGA-II and MNSGA-II}

This subsection shows the advantages of MNSGA-II over NSGA-II. The initial variables of both algorithms are the same. Unlike in single-objective optimization, there are two goals in a multiobjective optimization problem: (1) convergence to the Pareto-optimal set and (2) maintenance of diversity in solutions of the Pareto-optimal set. These two tasks cannot be measured adequately with one performance metric. Four examples are given including synchronization of the Rössler system with unconstrained $\Gamma$, synchronization of the hyperchaotic Rössler system with unconstrained $\Gamma$, synchronization of the Rössler system with constrained $\Gamma$, and synchronization of the hyperchaotic Rössler system with constrained $\Gamma$, which are investigated in Sec. IV B. The details of simulation results are given as follows.

\section{Synchronization of the Rössler system with unconstrained $\Gamma$}

The convergence process of the solutions obtained by NSGA-II and MNSGA-II with the generation number $T=80$ are shown in Fig. 5. It is revealed that MNSGA-II has the ability of appropriating Pareto front faster than NSGA-II. When $T=20$, some solutions generated by NSGA-II are dominated by the solutions of MNSGA-II. Some solutions obtained by MNSGA-II are discontinued and hence the diversity of the solutions is not satisfied. When $T=80$, a few solutions by NSGAII are still dominated by the solutions of MNSGA-II. The solutions obtained by MNSGA-II are averagely dispersed when the generation number $T$ increases. From our observations, one can see that although the diversity of the solutions obtained by both algorithms is similar after 80 generations, the MNSGA-II has a faster convergent speed compared with NSGA-II.

\section{Synchronization of the hyperchaotic Rössler system with unconstrained $\Gamma$}

The convergence process obtained by NSGA-II and MNSGA-II with the generation number $T=120$ is illustrated in Fig. 6. Similar with the results of synchronization of the Rössler with unconstrained $\Gamma$, it is shown from Fig. 6 that MNSGA-II is able to approach PF faster as with a NSGA-II as well as with a better diversity. After 20 generations, a few solutions generated by NSGA-II are dominated by the solutions of MNSGA-II and the diversity of solutions of NSGA-II

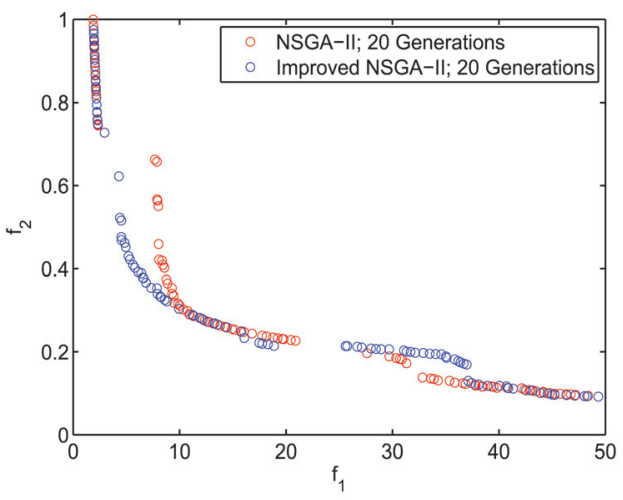

(a)

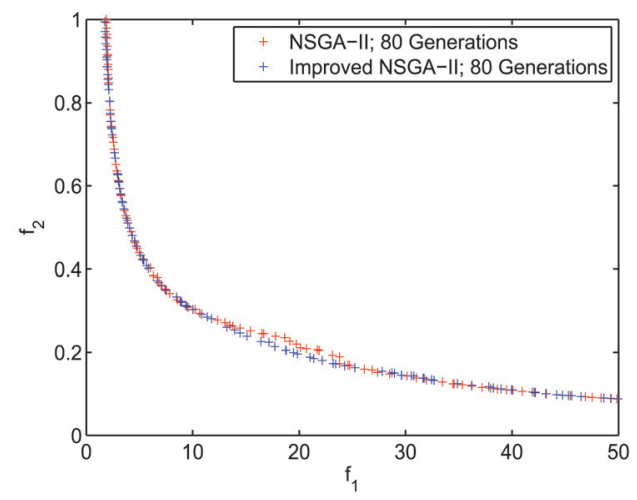

(b)
FIG. 5. (Color online) Convergence comparison of NSGA-II and MNSGA-II for the Rössler system with unconstrained $\Gamma$. 


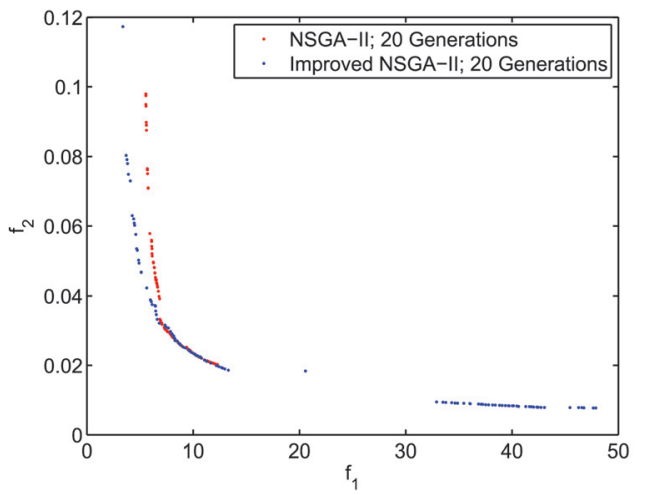

(a)

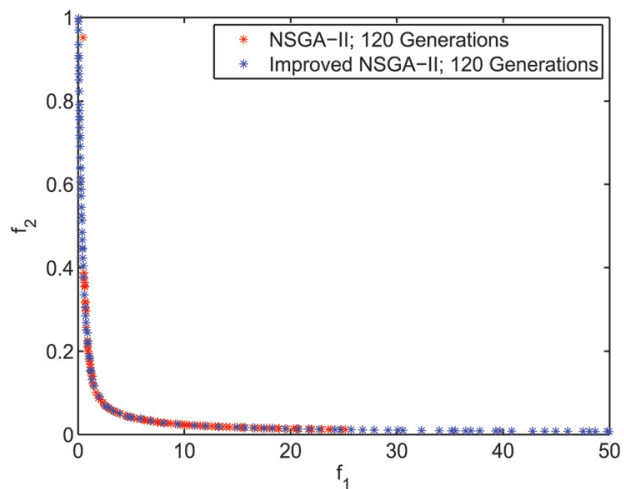

(b)
FIG. 6. (Color online) Convergence comparison of NSGA-II and MNSGA-II for the hyperchaotic Rössler system with unconstrained $\Gamma$. is poorer than that of MNSGA-II. After 120 generations, it is easily found that the convergence speed of MNSGA-II outperforms that of NSGA-II. After 120 generations, the solutions of MNSGA-II are averagely distributed in space, while the solutions of NSGA-II are a subset of the solutions of MSGA-II. Nearly a half of the solutions are not discovered by NSGA-II.

\section{Synchronization of the Rössler system with constrained $\Gamma$}

Fig. 7 shows the convergence process of the solutions obtained by NSGA-II and MNSGA-II with the generation number $T=120$. It can be seen from Figs. 7(a) and 7(b) that almost all the solutions obtained by NSGA-II are infeasible solutions although 20 steps are run. However, MNSGA-II has the ability of finding feasible solutions already after 20 steps only. Although the diversity of solutions obtained by MNSGA-II can be further improved, MNSGA-II outperforms NSGA-II much more in finding PF. After 120 generations, the solutions obtained by NSGA-II do not appear in the feasible space. However, the solutions generated by MNSGA-II converge to the PF. Therefore, NSGA-II lacks the ability of overcoming the constraints on $\Gamma$ and MNSGAII has the capability of finding feasible solutions.

\section{Synchronization of the hyperchaotic Rössler system with constrained $\Gamma$}

Fig. 8 depicts the convergent process of the solutions obtained by NSGA-II and MNSGA-II with the generation number $T=1000$. From Fig. 8(a), all the solutions obtained by NSGA-II are dominated by the solutions obtained by MNSGA-II after 200 generations. NSGA-II has a much slower convergence speed to approach PF than MNSGA-II does. On the other hand, the solutions obtained by MNSGAII are averagely dispersed in the feasible space. From Fig. 8(d), it can be seen that the solutions obtained by NSGA-II are a subset of those solutions obtained by NSGA-II. After 1000 generations, the solutions obtained by NSGA-II converge to the PF, which is also discovered by MNSGA-II.

In summary, MNSGA-II has a faster convergence speed of detecting PF than NSGA-II does, especially in dealing with the problems of synchronization of chaotic systems with constrained $\Gamma$. The solutions of MNSGA-II are also more diverse than those of NSGA-II after certain generations.

\section{Impact of the coupling strength on synchronization errors}

In this subsection, the impact of the coupling strength on synchronization errors is studied for DCNN. From Figs. 3 and 4 , it can be observed that the synchronization error for DCNN exhibit oscillations. For explaining this phenomenon, $K_{1}=\{26.3819, \quad 23.6171\}, \quad K_{2}=\{12.4238, \quad 8.3339\}$, $K_{3}=\{0.7708,0.0751\}$, and $\Gamma=\{1,1\}$ are chosen for synchronization of DCNN. The value of $K_{i}(i=1,2,3)$ is obtained by MNSGA-II. Only three representatives $K_{i}(i=1,2,3)$ are used here for the sake of simplicity. The synchronization errors are plotted (in $\log _{10}$ scale) in Fig. 9. It can be easily seen from Fig. 9 that with increasing the coupling $\operatorname{cost} C$, the

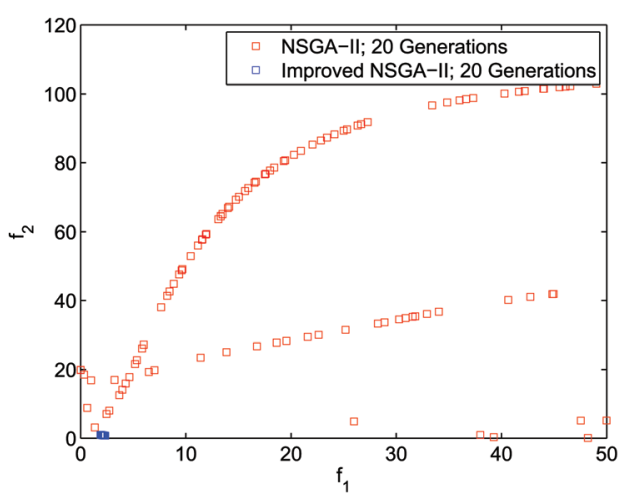

(a)

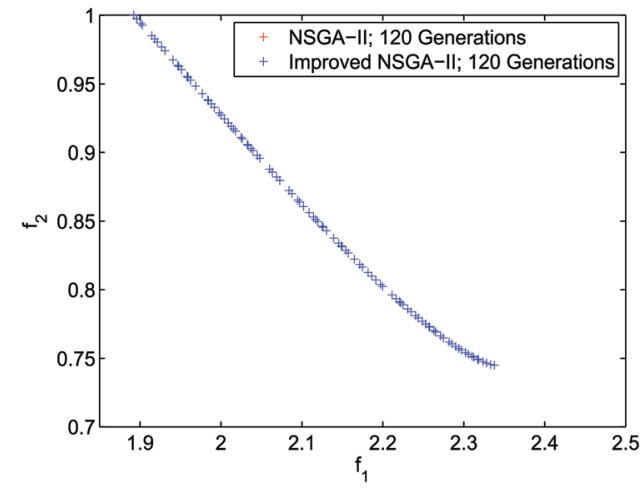

(b)
FIG. 7. (Color online) Convergence comparison of NSGA-II and MNSGA-II for the Rössler system with constrained $\Gamma$. 


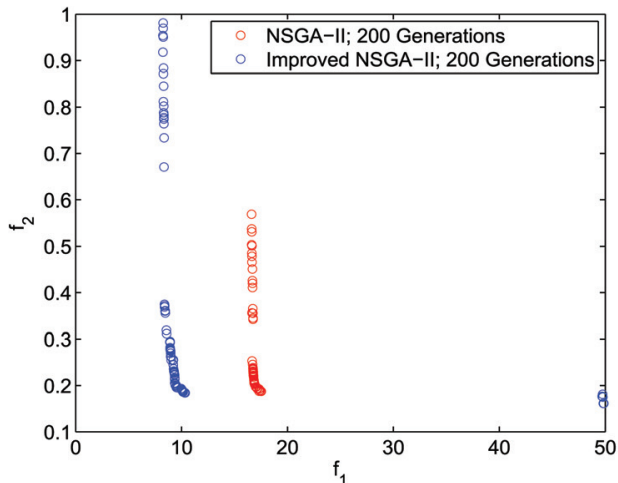

(a)

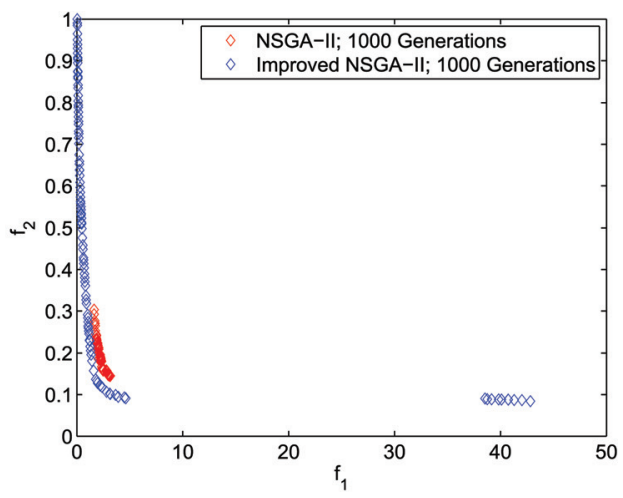

(b)
FIG. 8. (Color online) Convergence comparison of NSGA-II and MNSGA-II for the hyperchaotic Rössler system with constrained $\Gamma$. synchronization error has an increasing convergence speed. In addition, we find that the larger the coupling cost $C$, the more frequent or stronger peaks occur. From Fig. 9, one can also see that when using $K_{2}$ the frequency of peaks is the same as when using $K_{1}$. However, when using $K_{1}$ a larger amplitude of the peaks occurs than when using $K_{2}$. On the other hand, it can also be observed that the frequency of peaks when using $K_{2}$ is larger when using $K_{3}$, also with a larger amplitude of the peaks. These peaks/oscillations in this paper are due to the interplay between coupling strength and the individual dynamics. They do not only depend on the properties of the coupling strength, but also on the intrinsic properties of each individual dynamical system.

\section{E. Time complexity of NSGA-II and MNSGA-II}

The time complexity of NSGA-II and MNSGA-II is $O\left(M N^{2}\right)$, where $M$ is the objective number and $N$ is the population size. ${ }^{32}$ Actually, in real world application, the fitness evaluations of $f_{1}$ and $f_{2}$ overwhelm the algorithm. For example, when studying the problem of synchronization of the Rössler system with constrained $\Gamma$, the time of fitness evaluation occupies $90 \%$ of the total running time, and the time for carrying out the other part of the algorithm only costs $10 \%$ of the total running time.

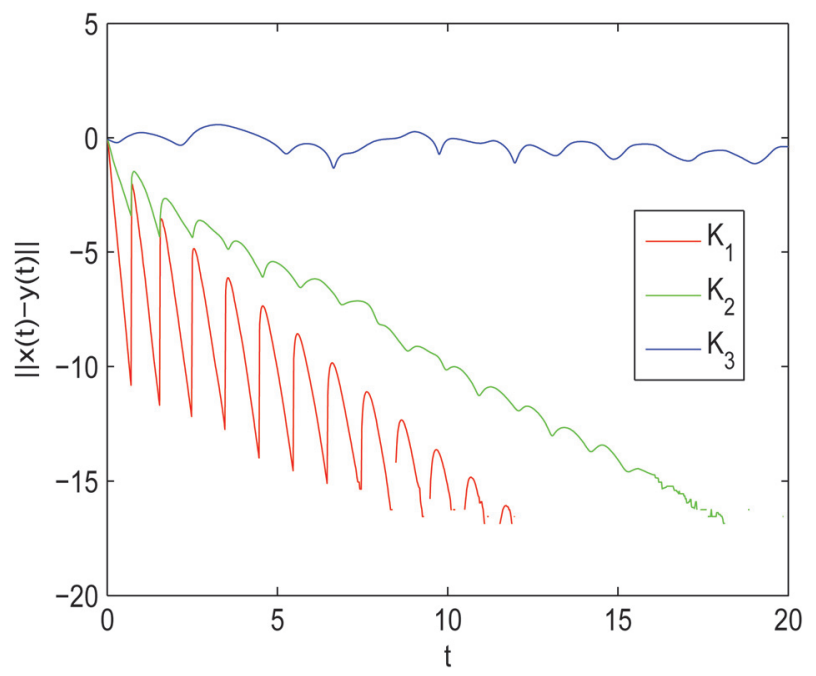

FIG. 9. (Color online) Synchronization error of DCNN when using different coupling strength.

\section{CONCLUSIONS}

In the field of designing coupling schemes of chaotic systems, one of the major challenges is to design optimal coupling strength to ensure synchronization of chaotic systems. In this paper, the problem of multiobjective synchronization problem of chaotic systems is investigated, in which both control cost of the coupling strength and convergence speed of synchronization are taken into consideration for optimization. In order to solve this multiobjective synchronization of chaotic systems with a fast convergence speed, an MNSGA-II is proposed by using a hybrid encoding scheme, which includes binary coding and real-coded coding schemes. The selections of appropriate coupling dimensions are also involved in the representations of MNSGA-II. Additionally, the comparisons of the adaptive updating method and NSGA-II algorithm with MNSGA-II are investigated in detail. The effectiveness of the MNSGA-II is examined by several numerical simulations.

Our approach can be extended and improved in several ways. On the one hand, the approach can be extended to study synchronization of chaotic systems with state switching, distributed delay, stochastic disturbances, and output coupling. One can utilize the proposed approach to obtain a better understanding of the interplay between the coupling dimensions and coupling strengths of different kinds of chaotic systems. It is also worth noting that the coupling strength is large; synchronization is dictated by the manner of driving the model with the data, so the estimation metric is small. When the coupling strength is too small, the data and the model do not synchronize, and information is not passed precisely between the data and the model. ${ }^{37}$ Therefore, how to properly design coupling strength is essential to achieve synchronization in coupled chaotic systems and estimation of unknown parameters of chaotic systems. Thus, an appropriate control gain can be designed according to the proposed method for identification of unknown parameters. On the other hand, since a number of solutions are displayed before a decision maker, in real world applications, the decision maker is not interested in the overall Pareto optimal front since the final decision is a unique solution. A simple and efficient way to solve the problem is to employ the recently developed reference point based technique, ${ }^{38}$ which might help to not only enhance the convergence speed of multiobjective evolutionary algorithm, but also provide a satisfactory solution to the decision maker. 


\section{ACKNOWLEDGMENTS}

The authors would like to express their sincere appreciation to the editor and reviewers for their helpful comments which help improve the presentation and quality of the paper.

The works of Y. Tang and W. K. Wong were supported by a research grant from The Hong Kong Polytechnic University (Project No. G-YH11). The work of Y. Tang was also supported by the Alexander von Humboldt Foundation of Germany. The work of Z. D. Wang was supported by the Engineering and Physical Sciences Research Council EPSRC of the U. K. under Grant No. GR/S27658/01, an International Joint Project sponsored by the Royal Society of the U.K., the International Science and Technology Cooperation Project of China (Grant No. 2009DFA32050), and the Alexander von Humboldt Foundation of Germany. The work of J. Kurths was supported by SUMO (EU) and ECONS (WGL). The work of J. A. Fang was supported by the National Natural Science Foundation of PR China (Grant No. 60874113), the Research Fund for the Doctoral Program of Higher Education (Grant No. 200802550007), the Key Creative Project of Shanghai Education Community (Grant No. 09ZZ66), the Key Foundation Project of Shanghai (Grant No. 09JC1400700).

${ }^{1}$ E. Ott, Chaos in Dynamical Systems, 2nd ed. (Cambridge University Press, Cambridge, England, 2002)

${ }^{2}$ I. I. Blekhman, Synchronization in Science and Technology (ASME, New York, 1988).

${ }^{3}$ L. M. Pecora and T. L. Carroll, Phys. Rev. Lett. 64, 821 (1990).

${ }^{4}$ U. Parlitz, Phys. Rev. Lett. 76, 1232 (1996).

${ }^{5}$ L. Kocarev and U. Parlitz, Phys. Rev. Lett. 76, 1816 (1996).

${ }^{6}$ N. Rulkov, M. Sushchik, L. Tsimring, and H. Abarbanel, Phys. Rev. E. 51, 980 (1995).

${ }^{7}$ A. Pikovsky, M. Rosenblum, and J. Kurths, Synchronization: A Universal Concept in Nonlinear Sciences (Cambridge University Press, Cambridge, England, 2001).

${ }^{8}$ Z. D. Wang, Y. Wang, and Y. Liu, IEEE Trans. Neural Networks 21, 11 (2010).
${ }^{9}$ T. Nishikawa and A. Motter, Proc. Natl. Acad. Sci. USA 107, 10342 (2010).

${ }^{10}$ L. Donetti, P. Hurtado, and M. Munoz, Phys. Rev. Lett. 95, 188701 (2005).

${ }^{11}$ C. Zhou, A. Motter, and J. Kurths, Phys. Rev. Lett. 96, 034101 (2006).

${ }^{12}$ Y. Tang, Z. D. Wang, and J. Fang, Chaos 19, 013112 (2009).

${ }^{13}$ S. Boccaletti, J. Kurths, G. Osipov, D. L. Valladares, and C. S. Zhou, Phys. Rep. 366, 1 (2002).

${ }^{14}$ Y. Tang, Z. D. Wang, and J. Fang, Information Sciences (unpublished).

${ }^{15}$ B. Ravoori, A. Cohen, A. Setty, F. Sorrentino, T. Murphy, E. Ott, and R. Roy, Phys. Rev. E 80, 056205 (2009).

${ }^{16}$ F. Sorrentino, Phys. Rev. E. 80, 056206 (2009).

${ }^{17}$ P. Lellis, M. Bernardo, and F. Garofalo, Chaos 18, 037110 (2008).

${ }^{18}$ D. Huang, Phys. Rev. Lett 93, 214101 (2004).

${ }^{19}$ C. Zhou and J. Kurths, Phys. Rev. Lett. 96, 164102 (2006).

${ }^{20}$ Y. Tang, H. Qiu, J. Fang, Q. Miao, and M. Xia, Phy. Lett. A 372, 4425 (2008).

${ }^{21}$ A. L. Fradkov and A. Y. Pogromsky, Introduction to Control of Oscillations and Chaos (World Scientific, Singapore, 1998).

${ }^{22}$ A. Fradkov and B. Andrievsky, Phys. Rev. E 78, 036210 (2008).

${ }^{23}$ H. Nijmeijer, Physica D 154, 219 (2001).

${ }^{24}$ R. Grigoriev, M. Cross, and H. Schuster, Phys. Rev. Lett. 79, 2795 (1997).

${ }^{25}$ X. Wang and G. Chen, Physica A 310, 521 (2002).

${ }^{26}$ A. Loria, IEEE Trans Circuits Syst., II: Express Briefs 56, 674 (2009).

${ }^{27} \mathrm{~K}$. Deb, Multi-Objective Optimization using Evolutionary Algorithms (Wiley, New York, 2002).

${ }^{28}$ D. E. Goldberg, Genetic Algorithms in Search, Optimization and Machine Learning (Addison-Wesley, Boston, MA, 1989).

${ }^{29}$ R. Krohling and J. Rey, IEEE Trans. Evol. Comput. 5, 78 (2001).

${ }^{30}$ M. Raymer, W. Punch, E. Goodman, L. Kuhn, and A. Jain, IEEE Trans. Evol. Comput. 4, 164 (2000).

${ }^{31}$ C. Goh, E. Teoh, and K. Tan, IEEE Trans. Neural Networks 19, 1531 (2008).

${ }^{32}$ K. Deb, A. Pratap, S. Agarwal, and T. Meyarivan, IEEE Trans. Evol. Comput. 6, 181 (2002).

${ }^{33}$ E. Zitzler, M. Laumanns, and L. Thiele, TIK-Report 103 (2001).

${ }^{34}$ O. Rossler, Phys. Lett. A 57, 397 (1976).

${ }^{35} \mathrm{H}$. Lu, Phys. Lett. A 298, 109 (2002).

${ }^{36}$ O. Rossler, Phys. Lett. A 71, 155 (1979).

${ }^{37}$ J. Quinn, P. Bryant, D. Creveling, S. Klein, and H. Abarbanel, Phys. Rev. E. 80, 016201 (2009).

${ }^{38}$ L. Said, S. Bechikh, and K. Ghédira, IEEE Trans. Evol. Comput. 14, 801 (2010). 